
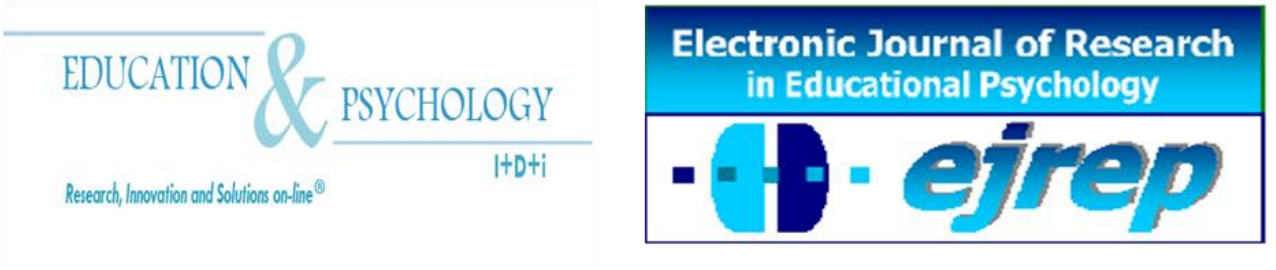

Editorial EOS

\title{
Personalidad, Procrastinación y Conducta Deshonesta en Alumnado de distintos Grados Universitarios
}

\author{
Mercè Clariana \\ Facultat de Psicologia, Universitat Autònoma de Barcelona, \\ $\mathrm{UAB}$, Barcelona
}

\section{España}

Correspondencia: Mercè Clariana. Facultat de Psicologia, Universitat Autònoma de Barcelona, 08193 Barcelona, Spain. E-mail: merce.clariana@uab.cat

(C) Education y Psychology I+D+i and Editorial EOS (Spain) 


\section{Resumen}

Introducción. La personalidad, la procrastinación y la conducta deshonesta en el aula (o cheating) son variables que han demostrado tener una influencia importante en el aprendizaje. Con todo, pocas veces se han estudiado todas juntas y menos teniendo también en cuenta el género del alumno y su elección de la carrera. Este trabajo analiza todas estas variables en estudiantes de distintas facultades de la Universitat Autònoma de Barcelona, UAB (Spain) con el objetivo de identificar diferencias y regularidades entre las titulaciones, que pueden ayudar a los responsables académicos a planificar los estudios para que puedan adaptarse mejor a las características de sus alumnos.

Método. Un total de 620 estudiantes de cuatro facultades de la UAB (Ingeniería, Economía, Humanidades y Educación) participaron voluntariamente en la investigación. Contestaron el cuestionario EDA, que evalúa procrastinación y cheating, y una versión S del Big Five Inventory, que mide personalidad. También participaron en una entrevista psicoeducativa individual que se utilizó para contrastar los resultados de los tests.

Resultados. Los resultados confirman la idea de que los alumnos de titulaciones universitarias distintas presentan características propias en cuanto a personalidad, procrastinación y cheating. Entre dichas peculiaridades cabe destacar que los alumnos de tecnología puntúan más alto en estabilidad emocional, y los de economía en procrastinación, y también que los estudiantes de educación obtienen notas más altas en consciencia y amabilidad que sus iguales de otras carreras. Todas estas relaciones están mediadas por el género del alumno que es una característica significativa a tener en cuenta en todas las variables estudiadas.

Discusión y Conclusiones. Los resultados de este trabajo amplían y matizan los que existían hasta ahora y permiten entender con más nitidez las relaciones que se establecen entre las características de los alumnos y las alumnas y las titulaciones que han elegido en la universidad.

Palabras Clave: elección de la carrera, RIASEC, grados universitarios, dimensiones de personalidad, procrastinación académica, conducta deshonesta en el aula o cheating.

Recibido: 24/03/13 Aceptación inicial: 02/07/13 Aceptación final: 08/07/13 


\title{
Personality, Procrastination and Cheating in Students from different University Degree Programs
}

\begin{abstract}
Introduction. Personality, procrastination and dishonest behaviour in the classroom (or cheating) are variables that have been seen to have an important influence on learning. However, they have seldom been studied together and even less taking into account the gender of the student and their choice of degree course. This work analyses the variables for students in different faculties of the Universitat Autònoma de Barcelona, UAB (Spain) with the aim of identifying similarities and differences between the different qualifications, which may help teaching staff to plan their courses so that they are better adapted to the characteristics of their students.
\end{abstract}

Method. A total of 620 students from four faculties of the UAB (Engineering, Economics, Humanities and Education) volunteered to take part in the research. The answered an EDA questionnaire to evaluate procrastination and cheating and an $\mathrm{S}$ version of the Big Five Inventory, which measures personality. They also took part in an individual educational psychology interview which was used to contrast the results of the tests.

Results. The results confirm the idea that students on different university degree courses have distinct characteristics in terms of personality, procrastination and cheating. Among the peculiarities is the face that technology students got higher scores for emotional stability and the economics students scored higher in procrastination. Education students got higher scores in awareness and kindness than their peers on other degree courses. All of these were mediated by the gender of the student which is a significant factor to take into account in all the variables being studied.

Discussion. The results of this work expand and clarify those that existed to date and give us a clearer understanding of the relations that are established between the students' characteristics and the university degree courses they choose.

Keywords: choice of degree course, RIASEC, university degrees, personality dimensions, academic procrastination, dishonest behaviour in the classroom or cheating.

Received: $\quad$ 03/24/13 Initial acceptance: $\quad$ 07/02/13 $\quad$ Final acceptance: 07/08/13 


\section{Introducción}

Las relaciones entre la personalidad y la elección de la carrera se han venido estudiando desde hace varios años. Algunos autores (Holland, 1985) aseguran que la plenitud profesional es el resultado de una buena correspondencia entre las características personales y la vocación, y otros, en la misma línea, defienden que una adecuada relación entre los rasgos de personalidad y el grado universitario elegido es la clave para conseguir el éxito, tanto en los estudios cómo en la vida laboral (Porter y Umbach, 2006).

Como consecuencia de éstos y otros trabajos, actualmente ya es posible establecer una conexión bastante firme entre la personalidad y la elección de la carrera. Así lo demuestran los resultados de algunos estudios publicados hasta ahora (Armstrong y Anthoney, 2009; DeFruyt y Mervielde, 1996; Donnay y Borgen, 1996; Tokar y Swanson, 1995), que informan, por ejemplo, de relaciones significativas entre los cinco factores de personalidad del Big Five (Costa y McCrae, 1992; Extraversión, Amabilidad, Consciencia, Neuroticismo y Apertura mental) y las dimensiones vocacionales del hexágono de Holland (1997), representadas por las siglas RIASEC, que corresponden, respectivamente a profesiones relacionadas con $\mathrm{R}=$ Realidad, I = Investigación, A = Arte, S = Sociedad, E = Empresa y C = actividades Convencionales o Conservadoras). La Figura 1 expone un diagrama sintético que puede ayudar a entender las conexiones que hasta ahora se han establecido entre la personalidad y la elección de la carrera.

Objetos

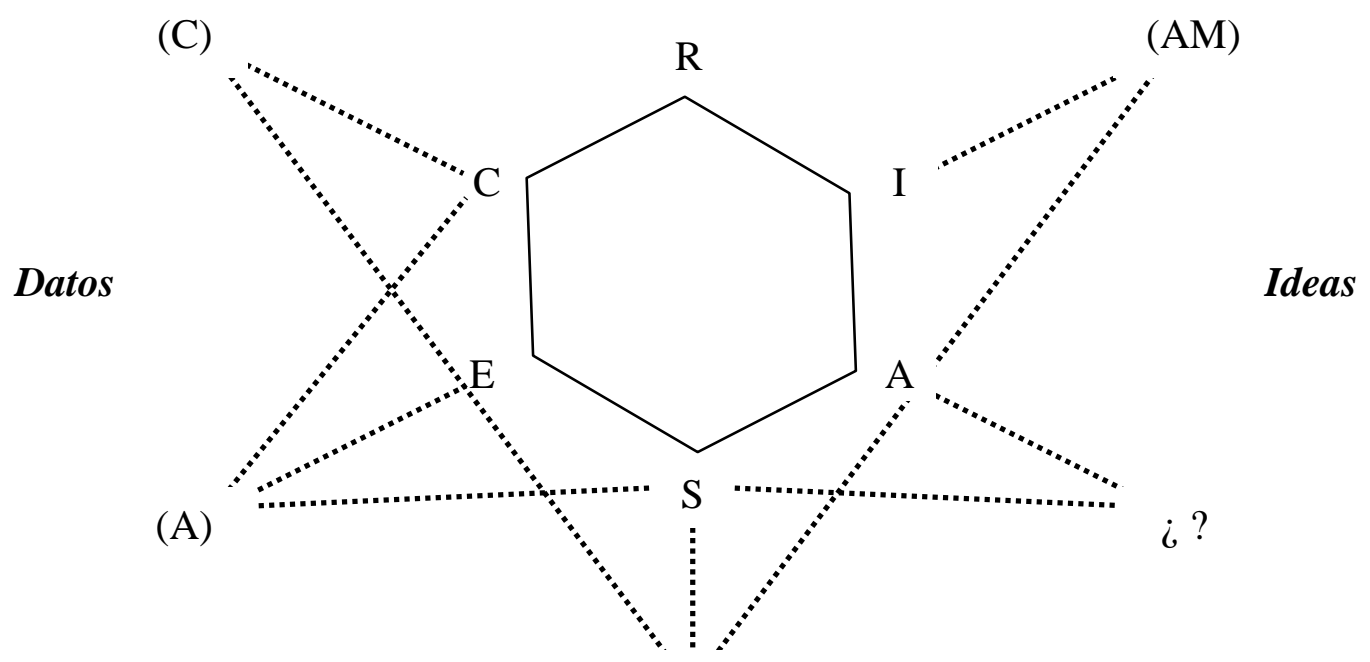

(E)

Personas

Figura 1. Diagrama hipotético de las relaciones previamente informadas entre las dimensiones de personalidad del Big Five (E) (A) (C) (N) (AM) y las preferencias vocacionales. 
Aparte de las referencias citadas hasta aquí, también abonan las relaciones dibujadas en la Figura 1 otros autores (por ejemplo, Hirschi, 2008), que indican la conexión entre personalidad y profesión sustentada en el siguiente patrón: las personas extravertidas se interesan por trabajos del eje artístico-social (en cursiva en el artículo original), las que puntúan alto en apertura mental se sienten atraídas por ocupaciones del eje realista-investigación y, finalmente, las que obtienen resultados elevados en amabilidad como dimensión de personalidad, tienen intereses profesionales centrados en el eje empresarial-convencional.

Con todo, y tal como se puede observar, la Figura 1 está todavía incompleta. Por ejemplo, no aclara qué dimensión o dimensiones de personalidad se relacionan con las actividades profesionales centradas en la Realidad, en parte porque éstas pueden ser muy variadas, cómo la física, el derecho o la biología (Holland, 1985). Además, las actividades de Investigación también cuestan de relacionar con rasgos de personalidad o carreras concretas, ya que adolecen del mismo problema. Al respecto de este punto, se ha afirmado (Amit y Sagiv, 2009) por ejemplo que "...investigation in physics is perceived as similar to Realistic occupations whereas (investigation) in history is perceived as similar to Artistic occupations" (p. 228). Asimismo, cabe añadir que hasta el momento no hay datos indicadores de la existencia de una relación específica entre el neuroticismo como factor de personalidad y las preferencias en el ámbito profesional. Es más, algunos expertos (Armstrong y Anthoney, 2009; DeFruyt y Mervielde, 1996) coinciden en señalar que esta característica de personalidad no está conectada con ninguna tendencia vocacional concreta, mientras que otros (Hirschi, 2008; Tokar y Swanson, 1995; Tokar, Fischer y Subich, 1998) afirman haber encontrado una débil conexión entre el neuroticismo y las actividades artísticas. Y para finalizar, también hay estudiosos que no ratifican las relaciones hasta aquí expuestas, y conectan por ejemplo, la apertura mental, con las ocupaciones empresariales (Zhao y Seibert, 2004), una relación que no hemos encontrado en ningún otro trabajo.

Además de estos resultados hay que añadir que otros autores han llamado la atención sobre les diferencias entre hombres y mujeres en la elección de la carrera, que también pueden ser significativas. En este sentido, se ha encontrado por ejemplo (Bethencourt y Cabrera Pérez, 2008) que los estudiantes de género masculino dan más importancia a las posibilidades laborales reales que tiene una especialidad concreta a la hora de escoger asignaturas en la uni- 
versidad, mientras que las alumnas consideran más sus intereses puramente vocacionales cuando hacen su elección.

Por otra parte, otros expertos se han dedicado a investigar las relaciones que se establecen entre la personalidad y dos características que influyen significativamente en los resultados académicos, cómo son la procrastinación, entendida cómo la tardanza innecesaria en la realización de las tareas (Schouwenburg y Lay, 1995), y la conducta deshonesta en el aula o cheating, que describe la tendencia a utilizar recursos no permitidos en el cumplimiento de las obligaciones en el ámbito de la educación formal (McCabe, Trevino y Butterfield, 2001).

Estas dos características influyen negativamente en el aprendizaje, pues se ha demostrado que la procrastinación, más común en los chicos que en las chicas (Steel, 2007; Steel y Ferrari, 2013), causa notas académicas bajas (Clariana, Gotzens, Badia y Cladellas, 2012; Wang y Englander, 2010), incrementa los sentimientos de debilidad e ineficacia en los alumnos que la practican (Surowiecki, 2010), y se asocia muy estrechamente a una falta de consciencia cómo dimensión de personalidad ( $r=$-.68; MacCann, Duckworth y Roberts, 2009; Steel, 2007, 2010; Steel y Ferrari, 2013). Este último es un aspecto muy a tener en cuenta, pues la consciencia ha demostrado correlacionar positivamente con buenos resultados académicos (Clariana, Gotzens y Badia, 2011; Meera, Karau, Schmeck y Avdic, 2011, entre muchos otros autores) y alta satisfacción en el estudio (Helmke y Schrader, 2000; Kuensting y Lipowsky, 2011).

A su vez, la investigación sobre cheating también ha aportado datos interesantes, tanto en relación a los resultados académicos como a la personalidad. En primer lugar, se confirma la hipótesis que indica que los alumnos son más deshonestos que las alumnas (Anderman y Murdock, 2007) y que los que practican cheating con frecuencia son más impulsivos que los puntuales (Anderman, Cupp y Lane, 2010) y obtienen peores notas, tanto en sus estudios (McCabe, 2009; McCabe, Trevino y Butterfield, 2001) como en la escala consciencia de los Big Five (Day, Hudson, Dobies y Waris, 2011; Yardley, Domènech, Bates y Nelson 2009). Por otra parte, también se ha comprobado que la procrastinación y el cheating van de la mano, pues se ha informado de correlaciones positivas y moderadamente altas entre las dos variables (Clariana et al., 2012; Roig y DeTommaso, 1995). Finalmente, cabe notar que hemos encontrado un trabajo que relaciona la conducta deshonesta en el aula con la elección de la carrera (Miller, Murdock, Anderman y Poindexter, 2007), y llega a la conclusión de que la tendencia 
a copiar es más frecuente en alumnos de ciencia y tecnología, y no tan extendida entre estudiantes de ciencias sociales, educación y humanidades.

Para acabar, también hay que señalar que algunos autores inciden en el hecho de que tanto la procrastinación cómo el cheating en el contexto educativo no son solamente características individuales o de personalidad, sino que también son resultado, en alguna medida, del tipo de prácticas instruccionales en que los alumnos participan (Roberts, 2008; Schouwenburg, Lay, Pychyl y Ferrari, 2004). Por ejemplo, está claro que los cursos que no contribuyen a motivar a los estudiantes, que son muy exigentes y no proporcionan la ayuda adecuada, que están a cargo de profesores incompetentes (Brent y Atkinson, 2011), o que dan instrucciones confusas sobre cómo hay que hacer el trabajo, provocan un aumento en la procrastinación del alumnado (Ackerman y Gross, 2005, 2007). En la misma línea, también se ha demostrado que la evaluación formativa y continua reduce significativamente la procrastinación y aumenta la satisfacción de los alumnos durante el estudio (Clariana, Gotzens y Badia, 2012).

Pero a pesar de estos avances, todavía son pocos los datos que pueden relacionar todas estas variables a la vez, la elección de la carrera, la personalidad, la procrastinación y el cheating. Además, las discrepancias que se dan en algunos de los resultados, que ya hemos mencionado, justifican también que se reconsidere de nuevo el tema.

\section{Objetivos}

Así pues, el objetivo del presente trabajo es ampliar la teoría existente, en dos sentidos:

1. Ampliar y puntualizar las relaciones encontradas por otros autores entre la personalidad y la elección de la carrera, que se han presentado en la Figura 1. Más concretamente, el trabajo pretende incluir en el modelo la diferenciación entre géneros, la o las dimensiones de personalidad que se relacionan con las preferencias vocacionales "Sociedad" y "Arte" de la teoría de Holland, y también relacionar las dimensiones de este autor con distintas titulaciones universitarias.

2. Incorporar al diagrama de la Figura 1 la procrastinación y el cheating en el ámbito académico. Tal como confirman algunos de los estudios que hemos citado, tanto la elección de la carrera como las características de personalidad pueden ser de ayuda para fijar dicha localización. 


\section{Método}

\section{Participantes}

Un total de 620 estudiantes voluntarios (59\% mujeres) de la Universitat Autònoma de Barcelona (UAB, España), un centro grande y público, participaron en la investigación. En el momento del estudio estaban matriculados en distintas titulaciones, que se pueden agrupar de acuerdo a la facultad que las imparte:

1. Ingeniería, que incluye grados de tecnología, matemáticas e informática ( $n=159 ; 27 \%$ mujeres). De ahora en adelante esta submuestra se llamará TEC.

2. Economía o negocios, una facultad que engloba titulaciones relacionadas con las ciencias empresariales y jurídicas, como empresariales, periodismo, derecho o sociología ( $n=168 ; 60 \%$ mujeres). Este grupo de alumnos se llamará BUS (de business).

3. La facultad de Educación, que comprende los grados de maestro y pedagogía ( $n=149$; $89 \%$ mujeres). Esta parte de la muestra se denominará EDU.

4. La facultad de Humanidades, que imparte grados de letras, geografía e historia $(n=$ 144; $62 \%$ mujeres). Para abreviar esta submuestra se denominará HUM.

Los estudiantes eran caucásicos y tenían de 17 a 30 años. El ANOVA calculado entre la edad de las cuatro submuestras $(F(3,616)=1.356, p=.255)$ no detecta diferencias significativas entre ellas. En cambio, la prueba de Ji cuadrado, $V$ de Cramer $=(3, n=620)=.443, p$ $<.001$, pone en evidencia que la distribución de sexos es muy desigual entre los cuatro grupos.

\section{Instrumentos}

\section{Entrevista psicoeducativa}

Los estudiantes participaron en una entrevista psicoeducativa a cargo de un alumno o alumna de último año de Psicología. La entrevista se utilizó para recoger información acerca de las habituales variables demográficas (edad, sexo, repetición de curso...), los resultados y satisfacción en el aprendizaje académico, en el pasado y en el momento actual, y las características individuales de los alumnos relativas a personalidad, procrastinación y cheating. En los casos en que había discrepancias significativas en estas últimas variables con los resultados de los tests, se omitieron los sujetos de la muestra. 


\section{Cuestionarios}

Además de la entrevista, se pidió a los alumnos que contestaran dos cuestionarios, garantizándoles en todo momento la preservación de su intimidad. Los cuestionarios utilizados en el estudio fueron:

1. El BFI10, que es una versión corta o S del Big Five Inventory, publicada por Rammstedt y John (2007). Esta escala utiliza 2 ítems por dimensión y en nuestra traducción obtuvo índices Alfa de Cronbach entre 0.62 (A) y 0.81 (E). En el estudio, para evitar confusiones, se utilizó el neuroticismo en sentido invertido, de modo que las puntuaciones altas en $\mathrm{N}$ indicaban buena estabilidad emocional. Algunos ejemplos de los ítems son: escala A, "Evito tener disputas con los demás"; escala E (ítem con puntuación inversa), "No me gusta hablar de mis cosas personales con otras personas". Las puntuaciones directas para todas las escalas de personalidad van de 2 a 10 puntos.

2. El EDA, que es un test factorial de dos dimensiones, procrastinación y cheating, desarrollado por Clariana y Martín (2008). La procrastinación se evalúa con 17 ítems y consiguió un Alfa de .91 en el presente estudio, y la conducta deshonesta en el aula se mide con 8 ítems que mostraron un Alfa de .82. Algunos de los reactivos son: escala de procrastinación, "Quiero ponerme a estudiar pero no encuentro el momento de empezar"; escala de cheating, "Copio los deberes de un compañero o de Internet". Las puntuaciones directas para procrastinación van de 17 a 85 y para cheating de 8 a 40 .

Todas las variables analizadas -personalidad E, A, C, N, AM, procrastinación y cheating- utilizaban una escala Likert de 5 puntos cómo respuesta a los ítems, con la puntuación 5 igual a "Totalmente de acuerdo" y la 1 igual a "Totalmente en desacuerdo". Igualmente, todas las escalas contenían ítems que puntuaban en sentido inverso.

\section{Análisis Estadístico}

Para los análisis estadísticos, la facultad a la que pertenecían los alumnos fue utilizada como variable indicadora de la selección de la carrera, con cuatro categorías: TEC, BUS, EDU y HUM. El género de los alumnos también se utilizó como variable categórica, en este caso con dos categorías: hombre y mujer. Y, por último, se utilizaron las 7 variables siguientes como continuas: las cinco escalas de personalidad E, A, C, N, AM, la procrastinación y el cheating. 
Se calcularon las correlaciones de Pearson entre las variables continuas para ver si se parecían a las anteriormente reportadas por otros autores. A continuación, se aplicó una prueba $t$ de Student de comparación de medias para datos independientes para comprobar las diferencias de género en las variables analizadas. _Para finalizar, y puesto que se supone y los trabajos presentados así lo demuestran, que las variables continuas del presente trabajo están relacionadas entre sí, para conocer cómo se relaciona la elección de la carrera con dichas variables se aplicó un análisis multivariante MANOVA.

\section{Resultados}

Las correlaciones entre las variables continuas estudiadas están expuestas en la Tabla 1. Como era de esperar la consciencia y la procrastinación están relacionadas muy estrechamente $(r=-.752)$, y también la consciencia con el cheating y la media de notas, aunque estas dos últimas correlaciones no son tan altas $(r=-.243$ y $r=.258$, respectivamente). Estos resultados son perfectamente acordes con estudios anteriores. Además, los datos revelan algunas relaciones inesperadas. Las más destacadas son la relación inversa que la amabilidad como factor de personalidad establece tanto con la procrastinación $(r=-.107)$ como con el cheating $(r=-.155)$, y también la relación directa que el cheating establece con la estabilidad emocional $(r=.126)$.

Tabla 1. Correlaciones bivariadas de Pearson entre las variables analizadas $(n=620)$

\begin{tabular}{lccccc}
\hline & \multicolumn{5}{c}{ Personalidad } \\
& $\mathrm{E}$ & $\mathrm{A}$ & $\mathrm{C}$ & $\mathrm{N}$ & $\mathrm{AM}$ \\
\hline Edad & -.011 & .002 & $.083^{*}$ & .065 & .072 \\
\hline Nota media & -.009 & -.009 & $.258^{* *}$ & -.077 & .034 \\
\hline Procrastinación & .011 & $-.107^{* *}$ & $-.752^{* *}$ & .094 & .021 \\
\hline Cheating & $.084^{*}$ & $-.155^{* *}$ & $-.243^{* *}$ & $.126^{* *}$ & -.018 \\
\hline
\end{tabular}

E = Extraversión, A = Amabilidad, C = Consciencia, N = Estabilidad emocional,

$\mathrm{AM}=$ Apertura mental.

* La correlación es significativa para $p<0.05$

** La correlación es significativa para $p<0.01$ 
A continuación se presentan los resultados de la $t$ de Student, en la Tabla 2. Cómo puede observarse, todas la variables continuas muestran diferencias significativas entre géneros menos la extraversión (E).

Tabla 2. Prueba t de Student entre géneros para las variables continuas analizadas (chicos: $n=255$; chicas: $n=365$ )

\begin{tabular}{|c|c|c|c|c|}
\hline $\begin{array}{l}\text { Variables } \\
\text { continuas }\end{array}$ & género & Media $(d t)$ & $t$ & $p$ \\
\hline \multirow{3}{*}{ Extraversión } & masculino & $6.52(2.16)$ & \multirow{3}{*}{-.49} & \multirow{3}{*}{.625} \\
\hline & & & & \\
\hline & femenino & $6.60(1.92)$ & & \\
\hline \multirow{3}{*}{ Amabilidad } & masculino & $5.52(1.62)$ & \multirow{3}{*}{-2.98} & \multirow{3}{*}{.003} \\
\hline & & & & \\
\hline & femenino & $5.59(1.82)$ & & \\
\hline \multirow{3}{*}{ Consciencia } & masculino & $5.71(1.99)$ & \multirow{3}{*}{$-.5 .62$} & \multirow{3}{*}{.000} \\
\hline & & & & \\
\hline & femenino & $6.65(2.06)$ & & \\
\hline \multirow{3}{*}{ Estabilidad emocional } & masculino & $6.46(1.83)$ & \multirow{3}{*}{10.46} & \multirow{3}{*}{.000} \\
\hline & & & & \\
\hline & femenino & $4.96(1.69)$ & & \\
\hline \multirow{3}{*}{ Apertura mental } & masculino & $7.66(1.71)$ & \multirow{3}{*}{6.00} & \multirow{3}{*}{.000} \\
\hline & & & & \\
\hline & femenino & $6.78(1.97)$ & & \\
\hline \multirow{3}{*}{ Procrastinación } & masculino & $51.23(12.53)$ & \multirow{3}{*}{5.44} & \multirow{3}{*}{.000} \\
\hline & & & & \\
\hline & femenino & $45.48(13.27)$ & & \\
\hline \multirow{3}{*}{ Cheating } & masculino & $20.31(5.54)$ & \multirow{3}{*}{3.80} & \multirow{3}{*}{.000} \\
\hline & & & & \\
\hline & femenino & $18.41(6.50)$ & & \\
\hline
\end{tabular}

Después se calculó el MANOVA. Todos los resultados de la prueba de Levene para las variables continuas alcanzaron una significación superior a .05. Esto indica que se puede asumir la igualdad de varianzas_por lo que, siguiendo a Pallant (2010) se utilizó la Lambda de Wilk en esta prueba. Bajo esta condición, el análisis arrojó un resultado de $F(7,610)=5.821$, $p<.001$, eta al cuadrado $=.063$. Puesto que la $p$ es inferior a .05 se puede afirmar que se han encontrado diferencias significativas entre las cuatro carreras, TEC, BUS, EDU y HUM, para todas las variables analizadas: E, A, C, N, AM, procrastinación y cheating. 
No obstante - y ya que la $t$ de Student ha puesto en evidencia que las diferencias de género en las variables estudiadas son significativas-, a continuación se han practicado dos MANOVAS más, uno para los hombres $(n=255)$ y otro para las mujeres $(n=365)$. Los detalles de estas pruebas están expuestos en las Tablas 3 y 4 en las cuales se puede observar, en primer lugar, que la significación del test de Levene para todas las variables continuas excepto una, la apertura mental en los hombres $(p<.001)$, sobrepasa el nivel del .05. Ya que esta condición se cumple mayoritariamente, se vuelve a aceptar la igualdad entre varianzas en los dos sexos para las variables estudiadas.

Tabla 3. MANOVA de las variables dependientes para las cuatro carreras: ingenierías TEC $(n=116)$, negocios BUS $(n=67)$, educación EDU $(n=17)$ y humanidades HUM $(n=55)$ :

Género masculino

\begin{tabular}{|c|c|c|c|c|c|}
\hline $\begin{array}{l}\text { Variables } \\
\text { continuas }\end{array}$ & $\begin{array}{c}\text { Significación } \\
\text { de Levene }\end{array}$ & $F(3,251)$ & Significación & $\begin{array}{c}\text { Eta al } \\
\text { cuadrado }\end{array}$ & Medias \\
\hline Extraversión & .359 & 1.288 & .279 & .015 & $\begin{array}{c}\text { TEC } 6.40 \\
\text { BUS } 6.91 \\
\text { EDU } 6.76 \\
\text { HUM } 6.22\end{array}$ \\
\hline Amabilidad & .358 & .346 & .792 & .004 & $\begin{array}{l}\text { TEC } 5.62 \\
\text { BUS } 5.51 \\
\text { EDU } 5.41 \\
\text { HUM } 5.36\end{array}$ \\
\hline Consciencia & .597 & .661 & .577 & .008 & $\begin{array}{c}\text { TEC } 5.58 \\
\text { BUS } 5.82 \\
\text { EDU } 5.41 \\
\text { HUM } 5.96\end{array}$ \\
\hline Estabilidad emocional & .590 & 2.438 & .065 & .028 & $\begin{array}{c}\text { TEC } 6.61^{*} \\
\text { BUS } 6.67^{*} \\
\text { EDU } 5.59 \\
\text { HUM } 6.15\end{array}$ \\
\hline Apertura mental & .001 & 2.727 & .045 & .032 & $\begin{array}{c}\text { TEC } 7.91^{*} \\
\text { BUS } 7.21 \\
\text { EDU } 7.35 \\
\text { HUM } 7.78^{*}\end{array}$ \\
\hline Procrastinacion & .241 & .575 & .632 & .007 & $\begin{array}{c}\text { TEC } 51.23 \\
\text { BUS } 52.45 \\
\text { EDU } 52.00 \\
\text { HUM } 49.51\end{array}$ \\
\hline
\end{tabular}




\begin{tabular}{lccccc}
\hline & & & TEC 20.48 \\
Cheating & .574 & .449 & .718 & .005 & BUS 20.67 \\
& & & & EDU 19.88 \\
& & & & HUM 19.52 \\
\hline
\end{tabular}

* Subsets significativamente altos de Tukey para $p<.05$

El MANOVA de los hombres presentaba una $F(7,245)=1.267, p=.189$, eta ${ }^{2}=.035$. Como puede observarse el resultado no es significativo, lo que indica que la elección de la carrera, en el género masculino, no es una variable que incida de una manera importante en los valores de los factores estudiados: personalidad, procrastinación y cheating. Los datos expuestos en la Tabla 3 confirman este hallazgo. De hecho, de las siete características analizadas se aprecian diferencias significativas únicamente en dos: la estabilidad emocional es más alta en los alumnos de tecnología y económicas y la apertura mental_da un valor significativamente alto en los alumnos de tecnología y humanidades. Además, el valor eta obtenido indica que la proporción de la varianza de las variables continuas que puede ser explicada por la variable categórica referida a la elección de la carrera, es francamente bajo en todos los casos, lo que confirma como ya hemos dicho que, para el grupo de estudiantes masculinos, las relaciones entre la elección de la carrera por un lado y la personalidad, la procrastinación y el cheating por el otro, son más bien débiles.

Tabla 4. MANOVA de las variables continuas para las cuatro carreras: ingenierías TEC $(n=43)$, negocios BUS $(n=101)$, educación EDU $(n=132)$ y humanidades HUM $(n=89)$ Género femenino

\begin{tabular}{|c|c|c|c|c|c|}
\hline $\begin{array}{l}\text { Variables } \\
\text { continuas }\end{array}$ & $\begin{array}{l}\text { Significación } \\
\text { de Levene }\end{array}$ & $F(3,361)$ & Significación & $\begin{array}{c}\text { Eta al } \\
\text { cuadrado }\end{array}$ & Medias \\
\hline Extraversión & .788 & .234 & .872 & .002 & $\begin{array}{c}\text { TEC } 6.51 \\
\text { BUS } 6.58 \\
\text { EDU } 6.70 \\
\text { HUM } 6.51\end{array}$ \\
\hline Amabilidad & .280 & 2.387 & .069 & .019 & $\begin{array}{r}\text { TEC } 5.79 \\
\text { BUS } 5.86 \\
\text { EDU } 6.27^{*} \\
\text { HUM 5.64 }\end{array}$ \\
\hline Consciencia & .823 & 4.164 & .006 & .033 & $\begin{array}{c}\text { TEC } 6.74 * \\
\text { BUS } 6.43 \\
\text { EDU } 7.10^{*} \\
\text { HUM } 6.18\end{array}$ \\
\hline
\end{tabular}




\begin{tabular}{|c|c|c|c|c|c|}
\hline Estabilidad emocional & .187 & 2.997 & .050 & .020 & $\begin{array}{c}\text { TEC } 4.60 \\
\text { BUS 5.32* } \\
\text { EDU } 4.81 \\
\text { HUM } 4.97\end{array}$ \\
\hline Apertura mental & .503 & 7.211 & .000 & .057 & $\begin{array}{c}\text { TEC } 7.33 * \\
\text { BUS } 6.57 \\
\text { EDU } 6.36 \\
\text { HUM } 7.37^{*}\end{array}$ \\
\hline Procrastinacion & .151 & 3.515 & .015 & .028 & $\begin{array}{c}\text { TEC } 44.49 \\
\text { BUS } 48.05^{*} \\
\text { EDU } 42.84 \\
\text { HUM } 46.94\end{array}$ \\
\hline Cheating & .067 & .907 & .438 & .007 & $\begin{array}{l}\text { TEC } 18.57 \\
\text { BUS } 19.19 \\
\text { EDU } 17.90 \\
\text { HUM } 18.30\end{array}$ \\
\hline
\end{tabular}

* Subsets significativamente altos de Tukey para $p<.05$

Por otro lado, el MANOVA para las mujeres arroja una $F(7,355)=2.435, p<.001$ eta al cuadrado $=.046$. En este caso el resultado sí es significativo y señala muchas más diferencias que las halladas en el otro género. Como puede observarse en la Tabla 4, en las mujeres hay muchas más variables que sí obtienen resultados significativamente distintos en función de la carrera que cursan las alumnas. El sentido de las diferencias indica que: las estudiantes de educación tienen notas altas en amabilidad y consciencia; las alumnas de tecnología y humanidades superan a las demás en apertura mental; además, las chicas que hacen tecnología también tienen notas significativamente más altas en consciencia; y finalmente las alumnas de negocios o derecho son las que muestran un resultado significativamente más alto tanto en estabilidad emocional como en procrastinación.

Para acabar, cabe añadir que la extraversión no ha resultado una variable diferenciadora ni para los hombres ni para las mujeres, y que ambos grupos y todas las titulaciones obtienen un resultado muy similar en esta característica.

\section{Discusión}

El objetivo del presente estudio era confirmar y ampliar la información sobre las relaciones que se establecen entre la elección de la carrera en base al modelo RIASEC (Holland, 
1985)-, las dimensiones E, A, C, N y AM de personalidad del Big Five (Costa y McCrae, 1992), la procrastinación y el cheating.

En primer lugar hay que señalar que algunas de las conexiones encontradas anteriormente se han replicado en el trabajo que ahora presentamos. Es el caso de la correlación alta y negativa entre consciencia y procrastinación, que se había fijado en $r=-.68$ (Steel, 2007) y que ahora ha resultado ser todavía más alta, de $r=-.75$; de la preferencia de las mujeres por carreras que implican trabajar con personas -educación y humanidades-, y de los hombres por ocupaciones que conllevan la relación con objetos o cosas -tecnología y empresariales(Holland, 1997), que se ha confirmado con la prueba de Ji cuadrado en nuestro caso; de que las estudiantes más puntuales, o menos procrastinadoras, son las de las carreras de ingeniería (Akinsola, Tella y Tella, 2007), aunque en el presente estudio no hemos podido demostrar esta afirmación para el género masculino; y, en la misma línea, también de la relación de la amabilidad y la consciencia con actividades profesionales de tipo convencional y social (Armstrong y Anthoney, 2009; DeFruyt y Mervielde, 1996; Tokar y Swanson, 1995), ya que el trabajo que ahora presentamos muestra que las alumnas que obtienen notas significativamente más altas en estas escalas son las que cursan estudios de las titulaciones de educación y del área tecnológica (ver Tabla 4, factor C consciencia).

Además, hay relaciones previamente establecidas que en esta ocasión no se han producido, como la tendencia de las personas extravertidas a escoger ocupaciones relacionadas con la asistencia a los demás o con las actividades empresariales (DeFruyt y Mervielde, 1996; Hirschi, 2008; Tokar y Swanson, 1995), ya que en nuestro estudio la extraversión no se ha relacionado significativamente con ninguna otra variable. Igualmente, la tendencia a copiar y al cheating, que estudios anteriores (Miller et al., 2007) habían conectado con carreras de ciencia y tecnologia, no se ha podido confirmar en el presente estudio (ver Tablas 3 y 4), que ha encontrado diferencias en esta variable en función de las titulaciones universitarias, aunque no significativas, y además a favor de los alumnos de derecho y empresariales, no de los de ciencia y tecnología.

Asimismo, respecto a la apertura mental, tenemos que decir que no se ha confirmado el miedo que expresan algunos autores, que dicen que el sistema educativo actual produce científicos que no tienen inteligencia ni creatividad porque excluye a los alumnos imaginativos para evitar problemas de rebelión e indisciplina, y aposta únicamente por los estudiantes 
que puntúan alto en consciencia y amabilidad. Como afirma Charlton (2009): "Creativity is probably associated with moderately high levels of Eysenck's personality trait of 'psychoticism'. Psychoticism combines qualities such as selfishness, independence from group norms, impulsivity and sensasion-seeking... But modern science selects for high conscientiousness and high agreeableness..." (p. 237). Aunque intuitivamente podríamos estar de acuerdo con este autor, tenemos que reconocer que, afortunadamente, los datos de la Tabla 4 no parecen apoyar esta afirmación por lo que respecta a las ciencias sociales y jurídicas. En cambio, es también notable el valor significativamente alto, al menos en las chicas, de la dimensión de personalidad consciencia en el área tecnológica.

Por lo demás, tampoco hemos confirmado algunos resultados anteriores (Lapan, Shaughnessy y Boggs, 1996) que decían, por un lado, que los sujetos más introvertidos escogen especialidades de matemáticas, pues en nuestro caso, como ya hemos indicado, la extraversión no ha sido una variable diferenciadora en ninguna de las carreras analizadas. Tampoco hemos ratificado que la tendencia a cometer cheating es más frecuente en alumnos de ciencia y tecnología (Miller et al., 2007), pues dicha tendencia no ha mostrado ninguna diferencia significativa entre carreras con nuestros datos, aunque sí lo ha hecho entre chicos y chicas.

Aparte de lo comentado hasta ahora, otras relaciones interesantes han aparecido en nuestro trabajo, que merece la pena hacer notar. Por un lado parece que los chicos son más estables emocionalmente que las chicas y que los que presentan esta característica escogen mayoritariamente carreras de ingeniería, económicas y derecho. También se ha puesto de manifiesto que las féminas que cursan especialidades de educación son significativamente distintas de todos los demás alumnos y alumnas, pues puntúan más alto en amabilidad y consciencia, dos características muy acordes con su futura profesión. Por último también nos han llamado la atención los resultados obtenidos con los alumnos de las carreras de económicas y derecho, pues por un lado ni los chicos ni las chicas obtienen puntuaciones significativamente altas en apertura mental como dimensión de personalidad, lo que es contradictorio con estudios anteriores (Zhao y Seibert, 2004), y por el otro los alumnos de business, periodismo y leyes del género masculino destacan significativamente en procrastinación, por encima de todos los demás grupos, lo que constituye realmente una novedad de este estudio (ver Tablas 3 y 4). 


\section{Objetos}

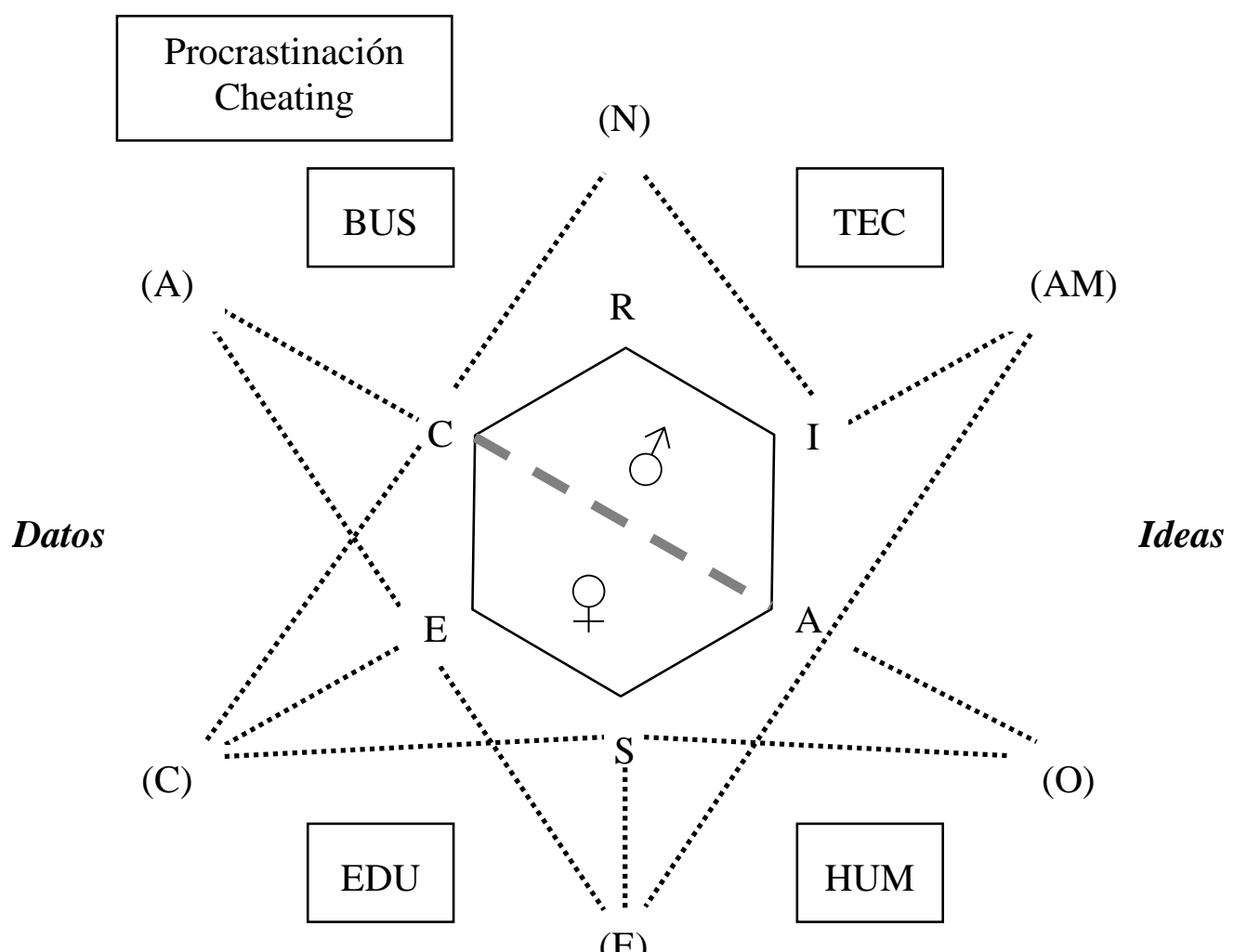

(E)

\section{Personas}

Figura 2. Diagrama, corregido a partir de los datos del presente estudio, de las relaciones entre las dimensiones de personalidad del Big Five (E) (A) (C) (N) (AM), las preferencias vocacionales de Holland RIASEC, la procrastinación académica y la conducta deshonesta en el aula o cheating

Con todo, lo que nos parece verdaderamente importante en nuestros resultados, y que ya hemos plasmado en la Figura 2, es la gran distancia entre géneros que hemos encontrado, tanto en la elección de la carrera como en las características específicas de personalidad, procrastinación y cheating que hemos analizado. Si bien es verdad que en la historia de la psicología se han puesto reiteradamente de manifiesto las diferencias entre géneros, en múltiples variables y aspectos (por ejemplo Bethencourt y Cabrera Pérez, 2008; Bubany y Hanse, 2011; de la Fuente Arias, 2004; DeAndrea, Carpenter, Shulman y Levine, 2009; deBruin y Hilton, 2007; García Berbén, 2005; Kuensting y Lipowsky, 2011; por citar solo algunos estudios relevantes del ámbito educativo) lo cierto es que en el siglo XXI, con una muestra acotada y depurada de universitarios, todos del mismo centro y de la misma edad, no esperábamos encontrar esta disimilitud tan relevante. Y es que, a nuestro entender, los resultados que presen- 
tamos dibujan dos tipos de estudiantes marcadamente distintos: por una parte están los chicos, con tendencia a la procrastinación y al cheating, con puntuaciones también altas en estabilidad emocional, y que escogen carreras próximas a las dimensiones CRIA de Holland, como son las de economía, derecho y tecnología. Por otra parte, en cambio, tenemos a las chicas, que procrastinan y engañan significativamente menos, que destacan en las dimensiones de personalidad de amabilidad y consciencia, y que prefieren estudiar carreras de la parte opuesta del hexágono de Holland, o sea relacionadas con las dimensiones CESA, como son las de educación y humanidades. No obstante esta división, por el momento bastante bien diferenciada, hay que hacer notar que hay un grupo numeroso de estudiantes, las chicas que se matriculan en empresariales, periodismo o derecho, que tienden a compartir características de ambos grupos, pues presentan una alta estabilidad emocional y puntuaciones también altas en procrastinación, igual que sus pares masculinos (de momento no encontramos más trabajos que confirmen esta afirmación, aunque queda bien establecida en el presente estudio)._En investigaciones posteriores, sin duda habrá que seguir a este subgrupo de intersección entre las particularidades académicas "masculinas" y las "femeninas", principalmente para comprobar si, con el paso del tiempo y la experiencia acumulada, estas chicas de empresariales y carreras relacionadas con el derecho y el mundo de la comunicación, se decantan por unas u otras características (de hombres o de mujeres), o bien se consolidan como un nuevo grupo de estudiantes con peculiaridades propias.

Y también como perspectiva de futuro, creemos que todavía hará falta volver a estudiar la personalidad, la procrastinación y el cheating con muestras más grandes y con más carreras universitarias, como las de biología y las de arte, las grandes ausentes en el presente trabajo por motivos ajenos a nuestra voluntad, siempre con la intención de ir completando el esquema que hemos elaborado para las Figuras que hemos presentado. En un tiempo hipotético en que todas las relaciones estén bien establecidas, igualmente con distinción de género si hace falta, seguramente los responsables académicos tendrán más facilidad para personalizar la acción educativa, un objetivo que de momento, es uno de los grandes retos que se plantea la educación universitaria.

\section{Agradecimientos}

La autora está en deuda con Nathalie Detmer, estudiante de la universidad de la Westfälische Wilhelms-Universität Münster (Alemania), por su ayuda en la recopilación de bibliografía y también por su entusiamo y optimismo en la realización del estudio. 


\section{Referencias}

Ackerman, D. S. y Gross, B. L. (2005). My instructor made me do it: Task characteristics and procrastination. Journal of Marketing education, 27, 5-13.

Ackerman, D. S. y Gross, B. L. (2007). I can start that JME manuscript next week, can’t I? The task characteristics behind why faculty procrastinate. Journal of Marketing education, $29,97-110$.

Akinsola, M. K., Tella, A. y Tella, A. (2007). Correlates of academic procrastination and mathematics achievement of university undergraduate students. Eurasia Journal of Mathematics, Science, and Technology, 3(4), 363-370.

Amit, A. y Sagiv, L. (2009). Where have the investigative occupations gone?: Perceptions and misperceptions of occupations. Journal of Career Assessment, 17(2), 214-231. doi: $10.1177 / 1069072708329032$

Anderman, E. M. y Murdoch, T. B. (2007). Psychology of Academic Cheating. Londres (UK): Elsevier.

Anderman, E. M., Cupp, P. K. y Lane, D. (2010). Psychology of Academic Cheating. Journal of Experimental Education, 78(1), 135-150.

Armstrong, P. I. y Anthoney, S. F. (2009). Personality facets and RIASEC interests: An integrated model Journal of Vocational Behavior, 75, 346-359. doi: 10.1016/j.jvb.2009.05.004

Bethencourt, J. T. y Cabrera, L. (2008). Comportamiento del alumnado universitario ante el desarrollo de su carrera profesional. Electronic Journal of Research in Educational Psychology, 16(6), 623-640.

Brent, E. y Atkisson, C. (2011). Accounting for cheating: An Evolving Theory and Emergent Themes. Research in Higher Education, 52 (6), 640-658. doi: 10.1007/s11162-010-9212-1

Bubany, S. T. y Hansen, J. C. (2011). Birth cohort change in the vocational interests of female and male college students. Journal of Vocational Behavior, 78 (1), 59-67. doi: 10.1016/j.jvb.2010.08.002

Charlton, B. G. (2009). Why are modern scientists so dull? How science selects for perseverance and sociability at the expense of intelligence and creativity. Medical Hypotheses, 72(3), 237-243.

Clariana, M. y Martín, M. (2008). Escala de Demora Académica (EDA). Revista de Psicología General y Aplicada, 61(1), 37-51.

Clariana, M., Gotzens, C. y Badia, M. (2011). Continuous assessment in a large group of psychology undergraduates. Electronic Journal of Research in Educational Psychology, 9(1), 95-112. 
Clariana, M., Gotzens, C. y Badia, M. (2012). Evaluación formativa y procrastinación en la universidad. Berlín: Lambert Academic Publishing LAP, Editorial Académica Española EAE.

Clariana, M., Gotzens, C., Badia, M. y Cladellas, R. (2012). Procrastination and cheating from secondary school to university. Electronic Journal of Research in Educational Psychology, 10(2), 737-754.

Costa, P. T. y McCrae, R. R. (1992). NEO PI-R Professional Manual. Odessa, FL: Psychological Assessment Resources.

Day, N. E., Hudson, D., Dobies, P. R. y Waris, R. (2011). Student or situation? Personality and classroom context as predictors of attitudes about business school cheating. Social Psychology of Education, 14(2), 261-282.

de la Fuente, J. (2004). Perspectivas recientes en el estudio de la motivación: la Teoría de la Orientación de Meta. Electronic Journal of Research in Educational Psychology, 2(1), $35-62$.

DeAndrea, D. C., Carpenter, C., Shulman, H. y Levine, T. R. (2009). The relationship between cheating behavior and sensation-seeking. Personality and Individual Differences, 47(8), 944-947. doi: 10.1016/j.paid.2009.07.021

deBruin, G. P. y Hilton, R. (2007). Examining the cheats: The role of conscientiousness and excitement seeking in academic dishonesty. South African Journal of Psychology, 37(1), $153-164$

DeFruyt, F. y Mervielde, I. (1996). Personality and interests as predictors of educational streaming and achievement. European Journal of Personality, 10, 405-425. doi: 0.1002/(SICI)1099-0984(199612)10:5<405::AID-PER255>3.0.CO;2-M

Donnay, D. A. D. y Borgen, F. H. (1996). Validity, structure, and content of the 1994 Strong Interest Inventory. Journal of Counseling Psychology, 43, 275-291. doi: 10.1037/00220167.43.3.275

García-Berbén, A. B. (2005). Estudio de los enfoques de aprendizaje en estudiantes de Magisterio y Psicopedagogía. Electronic Journal of Research in Educational Psychology, 3(2), 109-126.

Helmke, A. y Schrader, F. W. (2000). Procrastination im Studium. Erscheinungsformen und motivationale Bedingungen. En K. P. Wild y U. Schiefele (Eds.), Interesse und Lernmotivation. Untersuchungen zu Entwicklung, Förderung und Wirkung (pp. 207-225). Münster, GE: Waxmann. 
Hirschi, A. (2008). Personality complexes in adolescence: Traits, interests, work values, and self-evaluations. Personality and Individual Differences, 45(8), 716-721. doi: 10.1016/j.paid.2008.07.018

Holland, J. L. (1985). Vocational Preference Inventory Manual. Odessa, FL: Psychological Assessment Resources.

Holland, J. L. (1997). Making Vocational Choices: A Theory of Vocational Personalities and Work Environments. Odessa, FL: Psychological Assessment Resources ( $3^{\text {rd }} \mathrm{ed}$.).

Kuensting, J. y Lipowsky, F. (2011). Motivation for using a teacher education program and personality traits as predictors for study satisfaction and strategy use. Zeitschrift fur Padagogische Psychologie, 25(2), 105-114. doi: 10.1024/1010-0652/a000038

Lapan, R. T., Shaughnessy, P. y Boggs, K. (1996). Efficacy expectations and vocational interests as mediators between sex and choice of math/science college degrees: A longitudinal study. Journal of Vocational Behavior, 49, 277-291. doi: 10.1006/jvbe.1996.0044

MacCann, C., Duckworth, A. L. y Roberts, R. D. (2009). Empirical identification of the degree facets of conscientiousness. Learning and Individual Differences, 19(4), 451-458.

McCabe, D. L. (2009). Academic dishonesty in nursing schools: An empirical Investigation. Journal of Nursing Education, 48(11), 614-623. doi: 10.3928/01484834-20090716-07

McCabe, D. L., Trevino, L. K. y Butterfield, K. D. (2001). Cheating in academic institutions: A decade of research. Ethics \& Behavior, 11(3), 219-232. doi: 10.1207/S15327019EB1103_2

Meera, K., Karau, S. J., Schmeck, R. y Avdic, A. (2011). The Big Five personality traits, learning styles, and academic achievement. Personality and Individual Differences, 51 (4), 472-477. doi: 10.1016/j.paid.2011.04.019

Miller, A., D., Murdock, T. B., Anderman, E. M. y Poindexter, A. L. (2007). Who are all these cheaters? Characteristics of academically dishonest students. In E. Anderman y T. B. Murdoch (Eds.), Psychology of Academic Cheating (cap 2, pp. 9-32). London, UK: Elsevier.

Pallant, J. (2010). SPSS Survival Manual $4^{\text {th }}$ edition. New York, NY: Open University Press and McGraw-Hill education.

Porter, S. R. y Umbach, P. D. (2006). College degree choice: An analysis of the person environment fit. Research in Higher Education, 47(4), 429-449.

Rammstedt, B. y John, O. P. (2007). Measuring personality in one minute or less: A 10-item short version of the Big Five Inventory in English and German. Journal of Research in Personality, 41, 203-212. doi: 10.1016/j.jrp.2006.02.001 
Roberts, T. S. (2008). Student Plagiarism in an Online World. Nueva York (US): Information Science Reference.

Roig, M. y DeTommaso, L. (1995). Are college cheating and plagiarism related to academic procrastination? Psychological Reports, 77, 691-698.

Schouwenburg, H. C. y Lay, C. H. (1995). Trait procrastination and the Big-Five factors of personality. Personality and Individual Differences, 18, 481-490.

Schouwenburg, H. C., Lay, C. H., Pychyl, T. A. y Ferrari, J. R. (2004). Counseling the Procrastinator in Academic Settings. Washington DC (US): American Psychological Association APA.

Steel, P. (2007). The nature of procrastination: A meta-analytic and theoretical review of quintessential self-regulatory failure. Psychological Bulletin, 133, 65-94. doi: 10.1037/0033-2909.133.1.65

Steel, P. (2010). Arousal, avoidant and decisional procrastinators: Do they exist? Personality and Individual Differences, 48, 826-934. doi: 10.1016/j.paid.2010.02.025

Steel, P. y Ferrari, J. R. (2013). Sex, education and procrastination: An epidemiological study of procrastinator's characteristics from a global sample. European Journal of Personality, 27(1), 51-58. doi: 10.1002/per.1851

Surowiecki, (2010). Later. The New Yorker, 11 of October, http:/www.newyorker.com.

Tokar, D. M. y Swanson, J. L. (1995). Evaluation of the correspondence between Holland's vocational personality typology and the five-factor model of personality. Journal of Vocational Behavior, 46, 89-108. doi: 10.1006/jvbe.1995.1006

Tokar, D. M., Fischer, A. R. y Subich, L. M. (1998). Personality and vocational behavior: A selective review of the literature, 1993-1997. Journal of Vocational Behavior, 53, 115-153. doi: $10.1006 /$ jvbe. 1998.1660

Wang, Z. y Englander, F. (2010). A cross-disciplinary perspective on explaining student performance in introductory statistics. What is the relative impact of procrastination? College Student Journal, 44(2), 1-15. doi: 10.1006/drev.1996.0005

Yardley, J., Domènech, M., Bates, S. C. y Nelson, J. (2009). True confessions? Alumni’s retrospective reports on undergraduate cheating behaviors. Ethics \& Behavior, 19(1), 1-14.

Zhao, H. y Seibert, S. E. (2004). The big five personality dimensions and entrepreneurial status: A meta-analytical review. Journal of Applied Psychology, 91(2), 259-271. doi: $10.1037 / 0021-9010.91 .2 .259$ 